\title{
Glucose-targeted niosomes deliver vasoactive intestinal peptide (VIP) to the brain
}

\author{
Christine Dufes $^{\mathrm{a}, \mathrm{b}}$, Frederic Gaillard ${ }^{\mathrm{c}}$, Ijeoma F. Uchegbu ${ }^{\mathrm{d}}$, Andreas G. Schätzlein ${ }^{\mathrm{b}}$, \\ Jean-Christophe Olivier ${ }^{\mathrm{e}}$, Jean-Marc Muller ${ }^{\mathrm{a}, *}$ \\ a Equipe de Biologie des Interactions Cellulaires, LBSC CNRS UMR 6558, Faculté des Sciences, Universite de Poitiers, \\ 40 Avenue du Recteur Pineau, 86022 Poitiers Cedex, France \\ ${ }^{\mathrm{b}}$ Cancer Research UK Department of Medical Oncology, University of Glasgow, Garscube Estate, Switchback Road, \\ Glasgow G61 1BD, United Kingdom \\ ${ }^{c}$ Equipe du Développement Cortical, LBSC CNRS UMR 6558, Faculté des Sciences, Universite de Poitiers, \\ 40 Avenue du Recteur Pineau, 86022 Poitiers Cedex, France \\ ${ }^{\mathrm{d}}$ Department of Pharmaceutical Sciences, University of Strathclyde, Strathclyde Institute for Biomedical Sciences, \\ 27 Taylor Street, Glasgow G4 ONR, United Kingdom

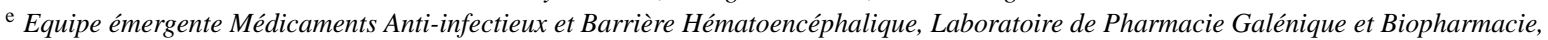 \\ Faculté de Médecine et Pharmacie, 34 rue du Jardin des Plantes, 86005 Poitiers Cedex, France
}

Received 13 July 2004; received in revised form 18 July 2004; accepted 19 July 2004

Available online 11 September 2004

\begin{abstract}
The aim of this study was to evaluate glucose-bearing niosomes as a brain targeted delivery system for the vasoactive intestinal peptide (VIP).

To this end, VIP/125I-VIP-loaded glucose-bearing niosomes were intravenously injected to mice. Brain uptake was determined by measuring the radioactivity of ${ }^{125}$ I-labeled VIP using $\gamma$-counting, after intravenous administration of VIP in solution or encapsulated in glucose-bearing niosomes or in control niosomes. VIP integrity was assessed by reversed-phase HPLC analysis of brain extracts. Distribution of ${ }^{125} \mathrm{I}-\mathrm{VIP}$ derived radioactivity was examined from serial brain slices.

HPLC analysis confirmed the presence of intact VIP in brain after administration of VIP-loaded niosomes, but not after administration of VIP solution. Encapsulation within glucose-bearing niosomes mainly allowed a significantly higher VIP brain uptake compared to control niosomes (up to $86 \%, 5$ min after treatment). Brain distribution of intact VIP after injection of glucose-bearing niosomes, indicated that radioactivity was preferentially located in the posterior and the anterior parts of the brain, whereas it was homogeneously distributed in the whole brain after the administration of control vesicles.
\end{abstract}

\footnotetext{
* Corresponding author. Tel.: +33 5494537 25; fax: +33 549453976.

E-mail address: Jean.Marc.Muller@univ-poitiers.fr (J.-M. Muller).
} 
In conclusion, this novel vesicular formulation of VIP delivers intact VIP to particular brain regions in mice. Glucose-bearing vesicles might be therefore a novel tool to deliver drugs across the blood-brain barrier (BBB).

(C) 2004 Elsevier B.V. All rights reserved.

Keywords: Niosomes; Glucose; Vasoactive intestinal peptide (VIP); Brain delivery; Blood-brain barrier

\section{Introduction}

The vasoactive intestinal peptide (VIP) is a 28-amino acid neuropeptide of the glucagon-secretin family (Mutt and Said, 1970) with a widespread distribution in both the central and peripheral nervous system (Fahrenkrug, 1979). It exerts diverse peripheral biological functions, such as anti-inflammatory and immunomodulatory effects, regulation of cell growth and differentiation and participation in the development of neural tissue (Muller et al., 1995). It also possesses neuroprotective properties that influence the survival of activity-dependent neurons in the central nervous system, and plays an important role in cerebral metabolism (Magistretti et al., 1981) and in cerebral carcinogenesis (Lelièvre et al., 1998). VIP may have interesting applications in the treatment of various neurological disorders: a lipophilic VIP analogue was proposed as a therapy against Alzheimer's disease (Gozes et al., 1996). However, like most endogenous peptides, its potential therapeutic applications are limited by its failure to cross the blood-brain barrier (BBB) which strictly controls the access of solutes to the brain (Begley, 1996) and by its rapid elimination after intravenous administration. Its blood half-life is less than $1 \mathrm{~min}$ in rat (Hassan et al., 1994) and approximately $1 \mathrm{~min}$ in man (Domschke et al., 1978). However, it has recently been shown that a weak amount of VIP appeared to reach the brain in conditions where a large amount of intravenous radiolabelled peptide was administrated (Dogrukol-Ak et al., 2003), which allowed to increase the sensitivity of the brain delivery assays utilized in these studies but also possible risks of side effects of this high concentration of free circulating radiopeptide.

In order to overcome this limitation, liposomal formulations for intravenous use have been proposed to protect VIP from degradation (Gao et al., 1994; Suzuki et al., 1996; Séjourné et al., 1997; Gololobov et al., 1998; Önyüksel et al., 1999). However, as liposomes were unable to cross the $\mathrm{BBB}$, the sole pharmacolog- ical effect obtained was a peripheral vasodilation. We recently delivered with success intact VIP to the brain in rats, by using an intranasal route of administration (Dufes et al., 2003). Through this pathway, VIP not only circumvents the BBB, but the polypeptide also avoids the hepatic first-pass effect and/or degradation in the blood compartment, a particularly important issue in the case of peptide drugs. Also for brain delivery, a VIP analogue was conjugated to the OX26 monoclonal murine antibody directed against the rat transferrin receptor (Bickel et al., 1993). This conjugation permitted a significant brain uptake of the analogue administered intravenously in rats, associated with a $65 \%$ increase in cerebral blood flow, compared to basal level (Bickel et al., 1993). Despite these attempts, no procedure promoting the passage of inherently nonpenetrating drugs through the intact brain blood vessel endothelium in their unmodified form is currently available.

Glucose analogues are theoretically good candidates for drug transport through the BBB. Indeed, the large and uninterrupted energetic demand of the brain is provided almost exclusively by $\beta$-D-glucose. Furthermore, the glucose consumption of the brain amounts to about $30 \%$ of the total body glucose consumption (Dick et al., 1984; La Manna and Harik, 1985). This high level of cerebral glucose uptake suggests that the facilitative $\beta$-D-glucose transporter GLUT1 might be a useful carrier for efficient and selective glucose-targeted drug delivery to the brain. Previous works using glucosechlorambucil derivatives and glycopeptide enkephalin analogues, as reported by Halmos et al. (1997) and Polt et al. (1994), respectively, indicate indeed that brain targeting through GLUT-1 mediated transport of glucose derivatives is possible. We hypothesized that VIP may reach the brain parenchyma in an intact form after encapsulation in a glucose-targeted system. To this end, we formulated VIP in glucose-targeted niosomes made from $N$-palmitoylglucosamine (Dufes et al., 2000), compared to the plain VIP niosomes and to the free drug. 


\section{Materials and methods}

\subsection{Chemicals}

$\mathrm{N}$-Palmitoylglucosamine (NPG) (Dufes et al., 2000) and ${ }^{125}$ I-VIP (Martin et al., 1986; Pineau et al., 2001) were synthesized and characterized as previously described. Human VIP was obtained from Neosystem (France). Bovine serum albumin (BSA), trifluoroacetic acid (TFA), sorbitan monostearate (Span 60) and cholesterol were all purchased from Sigma (France). Cholesteryl poly-24-oxyethylene ether (Solulan C24) was obtained from Amerchol (USA).

\subsection{Animals}

Male Swiss mice (30-40 g) obtained from Déprés Breeding Laboratories (St. Doulchard, France) were housed in the animal breeding facilities of the laboratory (Authorization no. 0028) for 4-6 days before experiments. They were maintained in a light-controlled (12/12 h light-dark cycle) and temperature-controlled environment with water and food ad libitum. Experimental procedures adhered to the Principles of Laboratory Animal Care (NIH Publication \# 86-23, revised 1985).

\subsection{Preparation of VIP loaded niosomes}

$N$-Palmitoylglucosamine (NPG) niosomes entrapping VIP were prepared by shaking a mixture of NPG (16 mg), Span 60 (65 mg), cholesterol (58 mg), Solulan $\mathrm{C} 24(54 \mathrm{mg})$ in PBS $(0.1 \mathrm{M}, \mathrm{pH}=7.4,1.5 \mathrm{~mL})$ at $90^{\circ} \mathrm{C}$ for $30 \mathrm{~min}$, followed by probe sonication (Soniprep 150, Fisher Scientific) for $5 \mathrm{~min}$ with the instrument set at $75 \%$ of its maximal capacity. The whole procedure of heating and sonication was repeated once. VIP was entrapped into niosomes by probe sonicating them in ${ }^{125} \mathrm{I}$-VIP $\left(5 \times 10^{6} \mathrm{cpm} / \mathrm{mL}\right.$ final concentration $)$ and unlabelled VIP $\left(10^{-9} \mathrm{~mol} / \mathrm{mL}\right.$ final concentration, prepared in PBS or in PBS supplemented with $1 \%(\mathrm{w} / \mathrm{v})$ BSA), in order to reduce the adsorption of VIP onto containers.

Control niosomes (without glucose) entrapping VIP were prepared in the same manner from a mixture of Span 60 (73 mg), cholesterol (65 mg), Solulan C24 $(54 \mathrm{mg})$ in PBS $(1.5 \mathrm{~mL})$ or in PBS supplemented with $1 \%(\mathrm{w} / \mathrm{v}) \mathrm{BSA})$.
To separate unencapsulated VIP from encapsulated VIP, the mixture was then centrifuged $(150,000 \times$ $g$, first for $1 \mathrm{~h}$, then for $30 \mathrm{~min}$, Optima LE-80K ultracentrifuge, Beckman Instruments, France) and the supernatant containing the unencapsulated VIP was discarded. After each ultracentrifugation step, the pelleted vesicles were resuspended in PBS $(2 \mathrm{~mL})$.

\subsection{Vesicle analysis}

\subsubsection{Assay for the amount of VIP entrapped by the vesicles}

Radioactivity in control or glucose-bearing vesicles was counted using a Cobra II Auto-Gamma Counting System (Packard, USA).

\subsubsection{Vesicle sizing}

Vesicle sizing was performed by photon correlation spectroscopy on a Malvern Zetasizer (Malvern Instruments, UK).

\subsection{Uptake of VIP entrapped in glucose-bearing vesicles in brain}

Mice ( $n=4$ animals/group) received three intravenous injections, at $5 \mathrm{~min}$ intervals, of VIP/ ${ }^{125} \mathrm{I}-\mathrm{VIP}$, free or encapsulated in glucose-bearing vesicles or in control vesicles. These formulations were administered at a dose equivalent to $6.6 \times 10^{-10} \mathrm{~mol} / \mathrm{kg}$ VIP $\left(100,000 \mathrm{cpm}{ }^{125} \mathrm{I}-\mathrm{VIP}\right.$ per $0.1 \mathrm{~mL}$ injection).

Five, 15 or $30 \mathrm{~min}$ after the last injection, the mice were sacrificed by cervical dislocation and a blood sample was collected by intracardiac puncture. A $200 \mathrm{~mL}$ volume of saline $(0.9 \% \mathrm{w} / \mathrm{v}$ sodium chloride) was then infused at room temperature into the clamped aorta to remove blood from brain vessels carefully. The brain was removed and weighed, and its radioactivity, as well as the radioactivity of the blood sample, was determined by $\gamma$-counting.

\subsection{HPLC analysis of intact VIP in the brain}

In an additional experiment the VIP integrity was examined by HPLC, after administration of VIP, free or entrapped in control or glucose-bearing niosomes.

Mice were dosed as above and sacrificed $15 \mathrm{~min}$ after the last administration. Brains were washed as described above, homogenized on ice with two 
volumes of $10 \mathrm{mM}$ Tris buffer ( $\mathrm{pH}$ 8) containing protease inhibitors $(1 \mathrm{mg} / \mathrm{mL}$ bacitracin, $1 \mathrm{mM}$ phenylmethylsulfonylfluoride, $0.2 \mathrm{mM}$ antipaïne and $2 \mathrm{mM}$ orthophenanthroline) using a Teflon-glass Potter tissue homogenizer and centrifuged at $4{ }^{\circ} \mathrm{C}(1,000 \times g$ for $10 \mathrm{~min}$ ). Supernatants were collected and stored on ice. The homogenization and centrifugation steps were applied two more times to the pellets. Finally, the three supernatants collected for each sample were pooled and ultracentrifuged at $100,000 \times g$ for $30 \mathrm{~min}$ at $4{ }^{\circ} \mathrm{C}$ to spin down any remaining tissue debris. The clear supernatant layers were collected and dried under vacuum using a Speed-Vac (Heto Maxi Dry Lyo FD1.0). Extracts were dissolved in $200 \mu \mathrm{L}$ mobile phase prior to HPLC analysis.

VIP reversed-phase HPLC was carried out using a $5 \mu \mathrm{m}$ VYDAC $\mathrm{C}_{18}$ column (Interchrom, France) with $\gamma$-counting of ${ }^{125} \mathrm{I}$-VIP. A two-step linear gradient was used with $0.1 \%(\mathrm{v} / \mathrm{v})$ TFA in water as solvent $\mathrm{A}$ and 85:15 acetonitrile: $0.1 \%(\mathrm{v} / \mathrm{v})$ TFA in water as solvent $\mathrm{B}$. The column was equilibrated with solvent $\mathrm{A}$ at a $1 \mathrm{~mL} / \mathrm{min}$ flow-rate. Injection volume was $100 \mu \mathrm{L}$. At each run, solvent B increased linearly from 0 to $35 \%$ in $2 \mathrm{~min}$, then up to $100 \%$ in further $15 \mathrm{~min}$, and was maintained at $100 \%$ for another $5 \mathrm{~min}$. Fractions of $1 \mathrm{~mL}$ were collected and $\gamma$-counted. VIP eluted in fractions $13-15$.

\subsection{Intracerebral distribution of VIP after administration in vesicles}

VIP formulations were administered as described above. Mice were anesthetized by intraperitoneal injection of $0.6 \%(\mathrm{w} / \mathrm{v})$ chloral hydrate $(300 \mathrm{mg} / \mathrm{kg}$ body weight) and perfused through the heart with $100 \mathrm{~mL}$ physiological saline followed by $200 \mathrm{~mL}$ fixative (4\% $\mathrm{w} / \mathrm{v}$ paraformaldehyde in saline). Brains were removed and cut serially ( $2.5 \mathrm{~mm}$-thick slices). Radioactivity was directly determined by $\gamma$-counting in each brain slices.

\subsection{Statistical analysis}

Results were expressed as means \pm S.E.M. Statistical significance was determined by one-way analysis of variance (ANOVA) followed by the Bonferroni's multiple comparison test. Differences were considered significant for $p<0.05$.

\section{Results}

\subsection{VIP is efficiently encapsulated into vesicles}

VIP was entrapped in the glucose-bearing vesicles $\left(3.03 \times 10^{-12} \pm 1.05 \times 10^{-13} \mathrm{~mol}\left(1.01 \times 10^{-8} \pm\right.\right.$ $3.51 \times 10^{-10} \mathrm{~g}$ ) per gram niosome, corresponding to $24.07 \pm 0.83 \%$ of the initial VIP) and in the control vesicles $\left(3.28 \times 10^{-12} \pm 7.27 \times 10^{-14} \mathrm{~mol}(1.09\right.$ $\times 10^{-8} \pm 2.42 \times 10^{-10} \mathrm{~g}$ ) per gram niosome, corresponding to $25.32 \pm 0.56 \%$ of the initial VIP). Control vesicles loaded with VIP had a $z$-average mean diameter of $214 \mathrm{~nm}$, while glucose vesicles loaded with VIP had a $z$-average mean diameter of $220 \mathrm{~nm}$.

\subsection{VIP entrapped in vesicles reaches the brain, and targeting with glucose improves the uptake}

No unexpected mortality of any animals occurred after administration of the VIP formulations used in the present study. As a result, vesicles were considered to be safe at the dosing schedule used. Radioactivity could be detected in the brain following the administration of glucose-bearing vesicles, indicating a passage of their content from the blood compartment into the brain parenchyma (Fig. 1). Brain radioactivity uptake was independent of the presence or not of BSA in the formulation: brain radioactivity level was 1.892 $\pm 329 \mathrm{cpm} / \mathrm{g}$ after administration of VIP vesicles prepared without BSA, not significantly different to 1.544 $\pm 325 \mathrm{cpm} / \mathrm{g}$ observed after administration of the vesicles prepared with this additive. The blood radioactivity

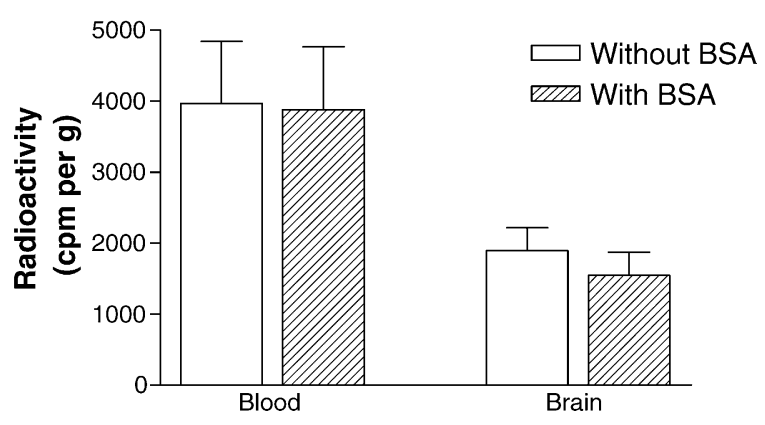

Fig. 1. Radioactivity uptake in the blood (left columns) and in the brain (right columns) after systemic administration of glucosebearing vesicles encapsulating VIP with or without BSA (four animals per formulation). 

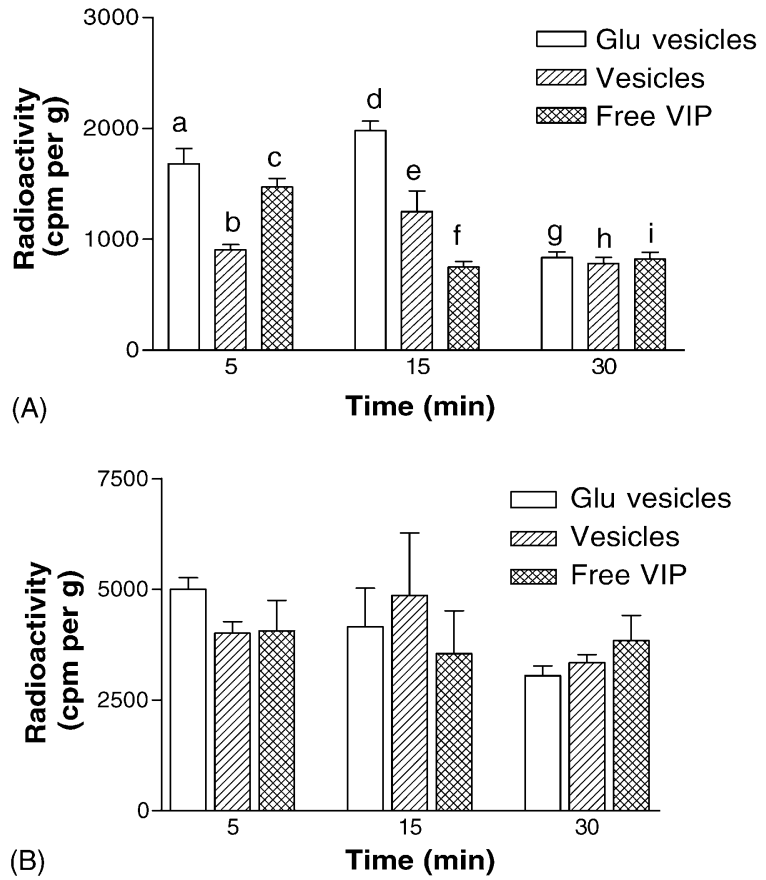

Fig. 2. Time-related radioactivity in brain (A) and blood (B) after systemic administrations of VIP encapsulated in glucose-bearing vesicles $(\mathrm{a}, \mathrm{d}, \mathrm{g})$; encapsulated in control vesicles $(\mathrm{b}, \mathrm{e}, \mathrm{h})$ and in solution (c, f, i) (four animals per formulation). Significant difference: a vs. (b, g), c vs. (b, f, i), d vs. (e, f). Blood radioactivity levels are not significantly different.

levels obtained after administration of these two formulations were also very similar (respectively $3.879 \pm$ $883 \mathrm{cpm} / \mathrm{g}$ and $3.966 \pm 876 \mathrm{cpm} / \mathrm{g}$ with and without BSA) (Fig. 1).

Brain and blood radioactivity was then measured at various time points after administration of VIP in different formulations (in solution, entrapped in glucose-bearing vesicles or in control vesicles, both of them prepared with BSA), as shown in Fig. 2. For vesicles prepared with and without glucose, the maximum brain uptake was reached 15 min post-administration (Fig. 2A). The glucose modified vesicles significantly increased brain radioactivity levels by comparison with control vesicles (by about $86 \%$ and $58 \%$ respectively $5 \mathrm{~min}$ and $15 \mathrm{~min}$ after treatment). With glucose-bearing vesicles, the brain radioactivity levels measured $15 \mathrm{~min}$ after the last intravenous injection represented $0.26 \% \pm 0.04 \%$ of the total radioactivity administered. After 30 min of administration, a reduced and similar residual level of radioactivity was found in the brain, as well in control (without glucose) or glucose-bearing vesicles. This might be due to a clearance (cerebrospinal fluid draining) or a degradation process limiting the amount of peptide inside the brain.

Regardless of the formulations, the blood radioactivity remained constant at each time point examined (Fig. 2B).

\subsection{Intact VIP is found in brain after administration in vesicles with BSA}

HPLC analysis performed on the brain extracts showed no peak corresponding to intact VIP after administration of glucose-bearing vesicles entrapping VIP without BSA (Fig. 3A). In contrast, a peak of radioactivity corresponding to intact VIP could be observed after administration of glucose-bearing vesicles entrapping VIP with BSA (Fig. 3B). Analysis also showed one peak corresponding to intact VIP as well after administration of vesicles without glucose but with BSA (Fig. 3C). The presence of BSA could decrease the interaction of VIP to lipids in the niosome membrane, thus allowing VIP to be released out of the vesicle.

In our HPLC experimental conditions, free radioiodine should elute very quickly, after few minutes of elution. Such early radioactive peak is not observed in experiments corresponding to glucose-bearing niosomes, indicating that radiolysis of the ${ }^{125}$ I-VIP does not occur in these experiments, at least for the fraction of radioligand that reached the brain.

No peak could be detected after administration of free VIP (Fig. 3D).

\subsection{Glucose-bearing vesicles target specific cerebral regions}

After administration of glucose-bearing vesicles, the radioactivity in the brain accumulated specifically in the posterior and the anterior parts of the brain (Fig. 4A). Counts for the posterior and the anterior sectors were respectively about two times and 1.5 times those read for the median sector or the cerebellum.

By contrast, no such pattern was observed following administration of VIP in control vesicles (Fig. 4B) or in solution (Fig. 4C). The distribution of radioactivity throughout the whole brain was homogenous. 

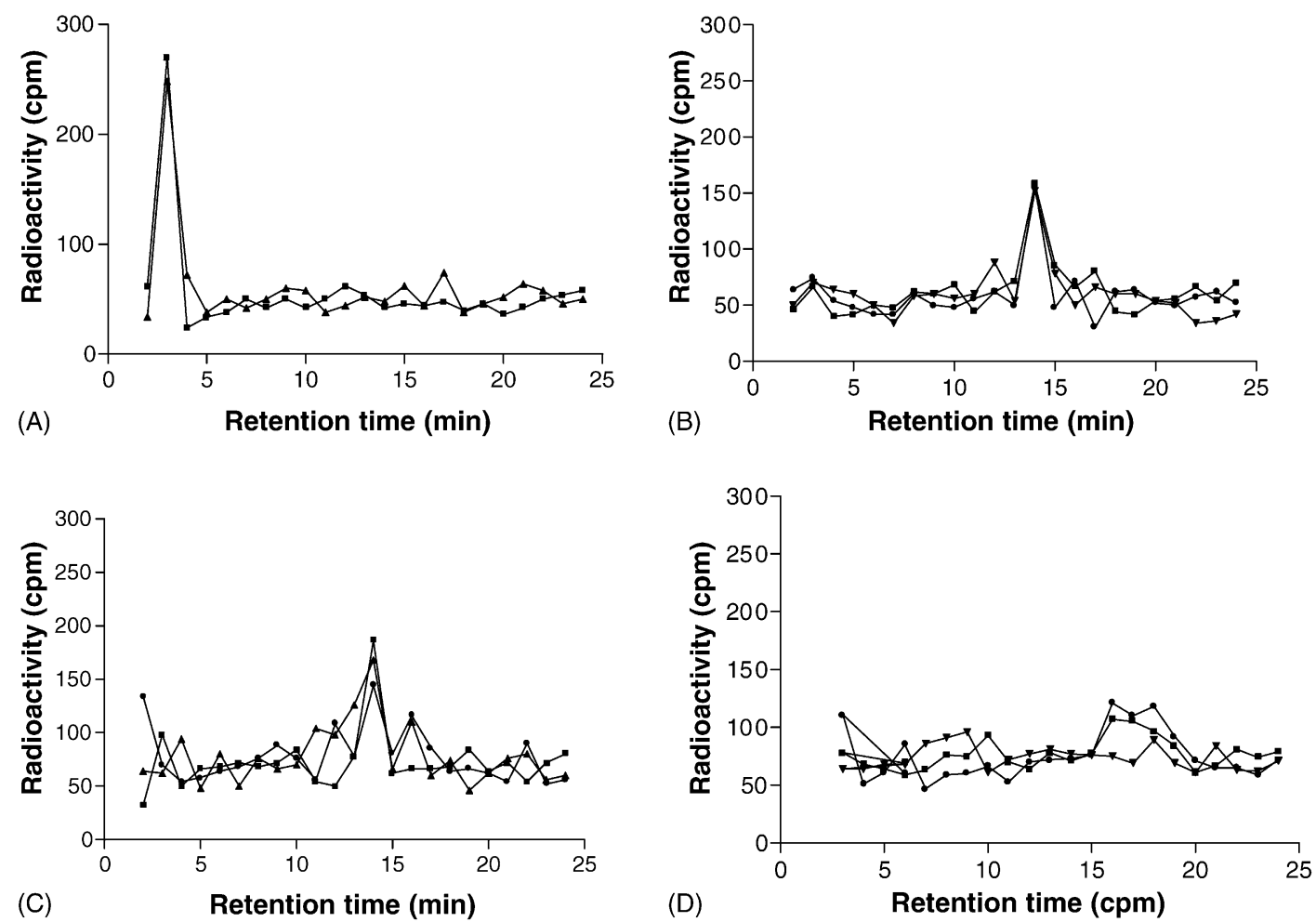

Fig. 3. Reversed-phase HPLC elution profiles of brain extracts obtained after intravenous administrations of VIP encapsulated in glucose-vesicles without BSA (A) or with BSA (B), VIP encapsulated in vesicles without glucose but with BSA (C) or as a solution (D), using ${ }^{125}$ I-VIP as a tracer (three animals per formulation). ${ }^{125} \mathrm{I}$-VIP was evaluated by $\gamma$-counting. Intact VIP eluted in fractions $13-15$. For more details see materials and methods.

\section{Discussion}

In a previous work (Dufes et al., 2000), we have reported the design and the characterization of glucosebearing niosomes, as well as their ability to bind to concanavalin A. The present study is an in vivo evaluation of these vesicles as a potential system to deliver the neuropeptide VIP into the brain.

There are three key findings of this study. First, we demonstrated that niosomes seem to be able to rapidly deliver intact VIP into brain provided that this neuropeptide is encapsulated in presence of BSA. Secondly, the presence of glucose ligands on the vesicles seems to improve this uptake, at least for the two first time points studied. Finally, only the administration of glucose-bearing vesicles induced a characteristic brain distribution of radioactivity, among which is intact VIP.
An important difficulty in CNS pharmacology is to elaborate tools able to overcome the limited penetration of peptidic drugs through the BBB. Our results suggest that VIP seems to be present as an intact molecule in cerebral extracts and in brain slices after systemic administration of niosomes encapsulating VIP. Although the mechanisms underlying stability and interaction of VIP in niosomes were not elucidated in this study, they might be related, in part, to the encapsulation of VIP in the vesicles' aqueous core. Such insertion might protect the peptide from degradation and might shift peptide conformation from random coil to helix, the optimal structure for VIP receptor interactions (Bodanszky et al., 1974; Robinson et al., 1982). To this end, Gao et al. (1994), Suzuki et al. (1996), Séjourné et al. (1997), Gololobov et al. (1998) and Önyüksel et al. (1999) showed that liposomal VIP was resistant to proteolytic cleavage, as a result of the impermeability 

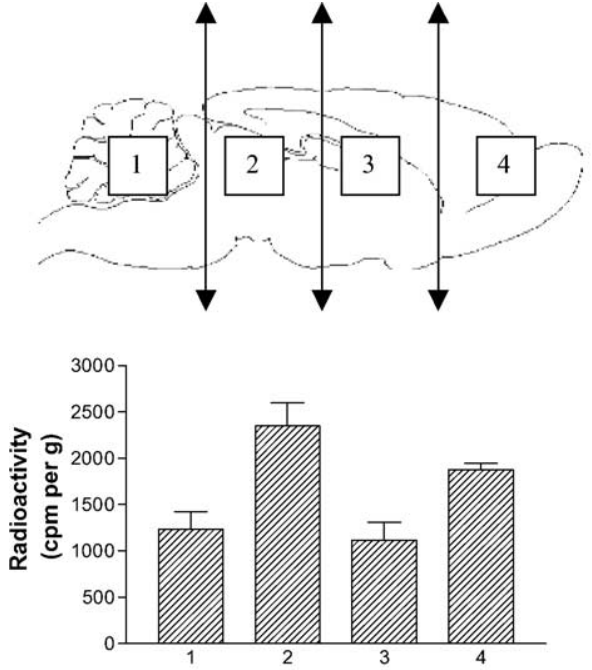

(A)

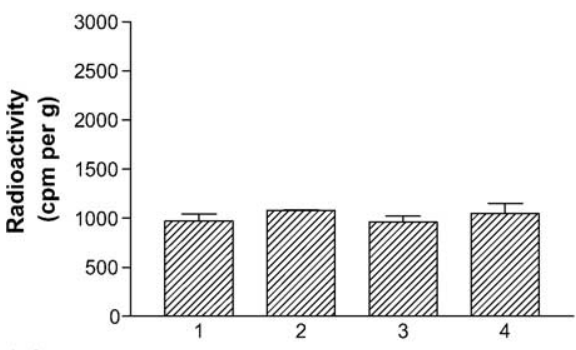

(B) Brain section number

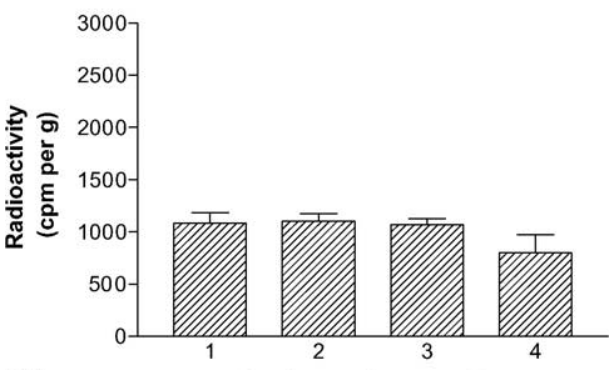

(C)

Brain section number

Fig. 4. Brain radioactivity distribution profiles after intravenous administration of VIP encapsulated in glucose-vesicles (A) or in vesicles without glucose (B), or free VIP (C), using ${ }^{125}$ I-VIP as a tracer (three animals per formulation). Radioactivity was evaluated by $\gamma$ counting of the $2.5-\mathrm{mm}$ thick brain slices. Arrows on the mouse brain schema denote the approximate spacing of the brain slices. For more details, see Section 2. In A, significant differences in radioactivity levels were found between Sections 1-4. Differences in B and C are not significant. of the vesicular bilayer to trypsin. In view of the ability of liposome constituents to interact with VIP, it is therefore hypothesized that VIP is "locked in" the vesicles by binding to lipids in the niosome membranes (Gololobov et al., 1998). The presence of BSA could decrease this interaction, thus allowing VIP to be released out of the vesicle.

When considering the uptake of radioactivity into the brain, the glucose modified vesicles led to significantly increased brain radioactivity levels compared to control vesicles (Fig. 2A). VIP degradation products, like other low molecular weight molecules, may enter cells by passive non-specific diffusion. High molecular weight carriers could eventually:

- be taken up via endocytosis, a comparatively slower but potentially more specific process, if their size allows this passage;

- or bind the BBB and release their content through the luminal membrane of the endothelial cells.

The glucose-targeted vesicles increased the brain uptake when compared to non-targeted vesicles.

The importance of carbohydrate-mediated recognition by specific cells has already been demonstrated. Umezawa and Eto (1988) reported that liposomes bearing aminophenyl mannoside were efficiently taken up into the mouse brain, suggesting that mannose could be recognized by the cells of the BBB. The BBB cells are known to express high levels of the glucose transporter GLUT1 (Dick et al., 1984). Therefore, an active targeting based on GLUT1 ligands could be a useful strategy for drug delivery in the brain. To our knowledge, our study is the first demonstration that VIP administered intravenously in targeted systems such as glucose-bearing niosomes is efficiently delivered to the brain.

Finally, we examined whether the different levels of brain radioactivity obtained after VIP administration through targeted or control vesicles display specific distributions. While control vesicles administration leads to an homogeneous distribution of radioactivity throughout the whole brain, the addition of glucose on the surface of the vesicles clearly modulated brain distribution of VIP derived radioactivity, among which was intact VIP (Fig. 4A). Since the glucose transporter GLUT1 present on the BBB cells may recognize $\beta$-D-glucose ligands of niosome surface, improvement may therefore be linked to a relatively higher glucose 
transporter concentration in some brain regions. This idea is supported by previous studies showing the presence of GLUT1 in considerable amounts throughout the brain, but with some regional variations (Brant et al., 1993; Rayner et al., 1994; Dobrogowska and Vorbrodt, 1999). Western blot studies revealed a relatively higher density in the posterior region of the brain such as the cerebellum (Brant et al., 1993; Rayner et al., 1994), whereas immunohistochemistry approaches indicated that GLUT1 was present in equal high amounts in the anterior cortex, the hippocampus and the cerebellum (Dobrogowska and Vorbrodt, 1999; Choeiri et al., 2002).

Moderate to low GLUT1 levels have been reported in the same studies (Dobrogowska and Vorbrodt, 1999; Choeiri et al., 2002) for the olfactory bulb, the striatum, the thalamus, the hypothalamus, the brainstem and the medulla. Interestingly, we observed high levels of radioactivity in Section 4 (including frontal, orbital and motor cortical areas) and Section 2 (including retrosplenial, visual and auditory cortices, hippocampus and midbrain). Sections with lower levels of radioactivity include brain regions containing lower densities of GLUT1 (striatum, thalamus, hypothalamus for Section 3; pons and medulla for Section 1). It should be noted in addition that orbital, retrosplenial, visual and auditory cortices exhibit significantly higher levels of glucose utilization (LCGU indices) than any other cortical area in the normal rat (Zilles and Wree, 1995). Together, high density of GLUT1 receptors and important rate of metabolic activity in these cortical areas could be responsible of the high VIP uptake observed in the anterior and posterior parts of the brain (Dobrogowska and Vorbrodt, 1999).

In conclusion, the present study demonstrated that the administration of systemic glucose-bearing vesicles encapsulating VIP could deliver intact VIP to specific brain areas. Therefore, glucose-bearing vesicles represent a novel tool to deliver drugs across the BBB.

\section{References}

Begley, D.J., 1996. The blood-brain barrier: principles for targeting peptides and drugs to the central nervous system. J. Pharm. Pharmacol. 48, 136-146.

Bickel, U., Yoshikawa, T., Landaw, E.M., Faull, K.F., Pardridge, W.M., 1993. Pharmacologic effects in vivo in brain by vector- mediated peptide drug delivery. Proc. Natl. Acad. Sci. U.S.A. 90, 2618-2622.

Bodanszky, M., Bodanszky, A., Klausner, Y.S., Said, S.I., 1974. A preferred conformation in the vasoactive intestinal peptide (VIP). Molecular architecture of gastrointestinal hormones. Bioorg. Chem. 3, 133-140.

Brant, A.M., Jess, T.J., Miligan, G., Brown, C.M., Gould, G.W., 1993. Immunological analysis of glucose transporters expressed in different regions of the rat brain and central nervous system. Biochem. Biophys. Res. Commun. 192, 1297-1302.

Choeiri, C., Staines, W., Messier, C., 2002. Immunohistochemical localization and quantification of glucose transporters in the mouse brain. Neuroscience 111, 19-34.

Dick, A.P.K., Harik, S.I., Walker, B.M., 1984. Identification and characterization of the glucose transporter of the blood-brain barrier by cytochalasin B binding and immunological reactivity. Proc. Natl. Acad. Sci. U.S.A. 81, 7233-7237.

Dobrogowska, D.H., Vorbrodt, A.W., 1999. Quantitative immunocytochemical study of blood-brain barrier glucose transporter (GLUT-1) in four regions of mouse brain. J. Histochem. Cytochem. 47, 1021-1029.

Dogrukol-Ak, D., Banks, A.W., Tuncel, N., Tuncel, M., 2003. Passage of vasoactive intestinal peptide across the blood-brain barrier. Peptides 24, 437-444.

Domschke, S., Domschke, W., Bloom, S.R., Mitznegg, P., Mitchell, S.J., Lux, G., Strunz, U., 1978. Vasoactive intestinal peptide in man: pharmacokinetics, metabolic and circulatory effects. Gut 19, 1049-1053.

Dufes, C., Schätzlein, A.G., Tetley, L., Gray, A.I., Watson, D.G., Olivier, J.C., Couet, W., Uchegbu, I.F., 2000. Niosomes and polymeric chitosan based vesicles bearing transferrin and glucose ligands for drug targeting. Pharm. Res. 17, 1250-1258.

Dufes, C., Olivier, J.C., Gaillard, F., Gaillard, A., Couet, W., Muller, J.M., 2003. Brain delivery of vasoactive intestinal peptide (VIP) following nasal administration to rats. Int. J. Pharm. 255, 87-97.

Fahrenkrug, J., 1979. Vasoactive intestinal polypeptide: measurement, distribution and putative neurotransmitter function. Digestion 19, 149-169.

Gao, X.P., Noda, Y., Rubinstein, I., Paul, S., 1994. Vasoactive intestinal peptide encapsulated in liposomes: effect on systemic arterial blood pressure. Life Sci. 54, PL247-PL252.

Gololobov, G., Noda, Y., Sherman, S., Rubinstein, I., BaranowskaKortylewicz, J., Paul, S., 1998. Stabilization of vasoactive intestinal peptide by lipids. J. Pharmacol. Exp. Ther. 285, 753-758.

Gozes, I., Bardea, A., Reshef, A., Zamostiano, R., Zhukovsky, S., Rubinraut, S., Fridkin, M., Brenneman, D.E., 1996. Neuroprotective strategy for Alzheimer disease: intranasal administration of a fatty neuropeptide. Proc. Natl. Acad. Sci. U.S.A. 93, 427-432.

Halmos, T., Santarromana, M., Antonakis, K., Scherman, D., 1997. Synthesis of glucose - chlorambucil derivatives and their recognition by the human Glut-1 glucose transporter. Eur. J. Pharmacol. 318, 477-484.

Hassan, M., Refai, E., Andersson, M., Schnell, P.O., Jacobsson, H., 1994. In vivo dynamical distribution of ${ }^{131}$ I-VIP in the rat studied by gamma-camera. Nucl. Med. Biol. 21, 865-872.

La Manna, J.C., Harik, S.I., 1985. Regional comparisons of brain glucose influx. Brain Res. 326, 299-305. 
Lelièvre, V., Pineau, N., Du, J., Wen, C.H., Nguyen, T., Janet, T., Muller, J.M., Waschek, J.A., 1998. Differential effects of peptide histidine isoleucine (PHI) and related peptides on stimulation and suppression of neuroblastoma cell proliferation. J. Biol. Chem. 273, 19685-19690.

Magistretti, P.J., Morrison, J.H., Shoemaker, W.J., Sapin, V., Bloom, F.E., 1981. Vasoactive intestinal polypeptide induces glycogenolysis in mouse cortical slices: a possible regulatory mechanism for the local control of energy metabolism. Proc. Natl. Acad. Sci. U.S.A. 78, 6535-6539.

Martin, J.L., Rose, K., Hughes, G.J., Magistretti, P.J., 1986. $\left[\right.$ Mono $\left[{ }^{125} \mathrm{I}\right]$ iodo-Tyr ${ }^{10}$-MetO ${ }^{17}$ ]-vasoactive intestinal polypeptide. Preparation, characterization, and use for radioimmunoassay and receptor binding. J. Biol. Chem. 25, 5320-5327.

Muller, J.M., Lelievre, V., Becq-Giraudon, L., Meunier, A.C., 1995. VIP as a cell-growth and differentiation neuromodulator role in neurodevelopment. Mol. Neurobiol. 10, 115-134.

Mutt, V., Said, S.I., 1970. Polypeptide with broad biological activity: isolation from small intestine. Science 169, 1217-1218.

Önyüksel, H., Ikezaki, H., Patel, M., Gao, X.P., Rubinstein, I., 1999. A novel formulation of VIP in sterically stabilized micelles amplifies vasodilation in vivo. Pharm. Res. 16, 155-160.

Pineau, N., Lelievre, V., Goursaud, S., Hilairet, S., Waschek, J.A., Janet, T., Muller, J.M., 2001. The polypeptide PHI discriminates a GTP-insensitive form of VIP receptor in liver membranes. Neuropeptides $35,1-10$.
Polt, R., Porreca, F., Szabo, L.Z., Bilsky, A.J., Davis, P., Albruscato, T.J., Davis, T.P., Horvath, R., Yamamura, H.I., Hruby, V.J., 1994. Glycopeptide enkephalin analogues produce analgesia in mice: evidence for penetration of the blood-brain barrier. Proc. Natl. Acad. Sci. U.S.A. 91, 7114-7118.

Rayner, D.V., Thomas, M.E., Trayhurn, P., 1994. Glucose transporters (GLUTs 1-4) and their mRNAs in regions of the rat brain: insulin-sensitive transporter expression in the cerebellum. Can. Pharmacol. J. 72, 476-479.

Robinson, R.M., Blakeney Jr., E.W., Mattice, W.L., 1982. Lipidinduced conformational changes in glucagon, secretin and vasoactive intestinal peptide. Biopolymers 21, 1217-1228.

Séjourné, F., Rubinstein, I., Suzuki, H., Alkan-Önyüksel, H., 1997. Development of a novel bioactive formulation of vasoactive intestinal peptide in sterically stabilized liposomes. Pharm. Res. 14, 362-365.

Suzuki, H., Noda, Y., Gao, X.P., Séjourné, F., Alkan-Önyüksen, H., Paul, S., Rubinstein, I., 1996. Encapsulation of VIP into liposomes restores vasorelaxation in hypertension in situ. Am. J. Physiol. 271, H282-H287.

Umezawa, F., Eto, Y., 1988. Liposome targeting to mouse brain: mannose as a recognition marker. Biochem. Biophys. Res. Commun. 153, 1038-1044.

Zilles, K., Wree, A., 1995. Cortex: areal and laminar structures. In: Paxinos, G. (Ed.), The Rat Nervous System, 2nd edition. Academic Press, Sydney, pp. 649-685. 